\title{
Universal Introduction to the Quantum Hall Effect
}

\author{
Keshav N. Shrivastava, Senior Member, IACSIT
}

\begin{abstract}
The Hall effect is the generation of a current perpendicular to the direction of applied electric as well as applied magnetic field in a metal or a semiconductor. It is used to determine the concentration of electrons. The Quantum Hall effect has been discovered by von Klitzing in Germany and by Tsui, Stormer and Gossard in U.S.A. Robert Laughlin also in U.S.A. explained the quantization of Hall current by using "flux quantization" and introduced incompressibility to obtain fractional charge. We have developed the theory of the quantum Hall effect by using the theory of angular momentum. Our predicted fractions are in accord with those measured. Von Klitzing in 1985, and Tsui, Stormer and Laughlin in 1998 received the Nobel prize for their discoveries. In this lecture, we report a review of the subject as well as emphasize our explanation of the observed phenomena. We use spin to explain the fractional charge and hence we discover spin-charge coupling. We find a new way to quantize the flux and compare our explanation with other prize winning researches.
\end{abstract}

Index Terms-Angular momentum, flux quantization, hall effect, modified landau levels, modified lande's g value formula.

\section{INTRODUCTION}

The ordinary Hall effect was discovered by Edwin Hall [1] in 1879. In 1930, Landau showed that the orbital motion of the electron gives magnetic susceptibility. In $1978 \mathrm{~K}$. von Klitzing and Th. Englert [2] found a plateau in the Hall effect. In 1980 von Klitzing et al. [3] found the value of $\mathrm{h} / \mathrm{e}^{2}$ from the plateau in the Hall effect. In 1985, Klitzing was awarded the Nobel prize in Physics for the discovery of quantum Hall effect. In 1982, D. C. Tsui, H. L. Stormer and A. C. Gossard [4] discovered the steps at fractional numbers which was extended by Willet et al. [5]. Laughlin wrote a wave function which gave the ideas of a fractional charge [6]. Shrivastava [7] wrote the correct theory which agrees with the experimental data. In 1998, Tsui, Stormer and Laughlin were awarded the Nobel prize.

\section{A. Applications of the Hall Effect}

1) Hall probe. The detection of magnetic fields is often done by using Hall currents.

2) Hall effect sensors. The sensor responds to changes in the magnetic field intensity.

3) Hall effect motors and switches.

The force is $\boldsymbol{F}=e \boldsymbol{v} x \boldsymbol{B}$ so that the Hall voltage is, $\mathrm{V}=$ $\mathrm{IB} /$ necd

where $I$ is the Hall current, B is the magnetic induction, $\mathrm{n}$ the electron concentration, $\mathrm{e}$ is the electron charge, $\mathrm{c}$ is the velocity of light and $d$ is the thickness of the sample as shown

Manuscript received February 7, 2013; revise March 22, 2013.

K. N. Shrivastava is with the University of Hyderabad, Hyderabad 500046, India (e-mail: keshav1001@yahoo.com). in the Fig. 1.

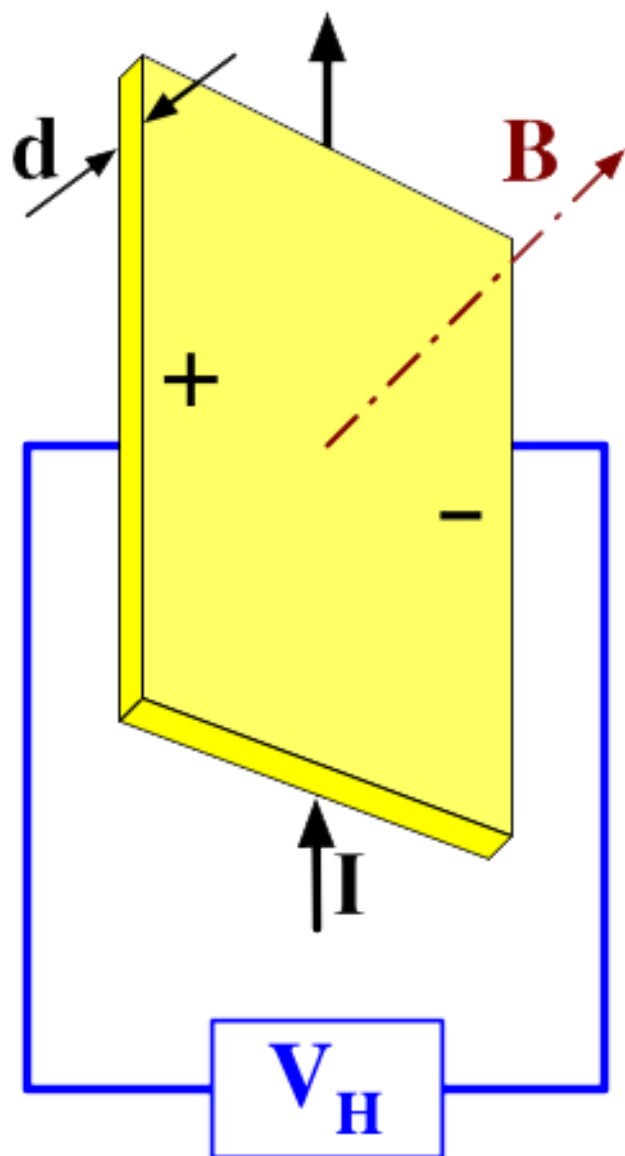

Fig. 1. The Hall voltage is measured orthogonal to both the applied electric as well as the magnetic field.

\section{B. Two Dimensional Electron Systems}

A two dimensional electron system is formed in a heterostructure which has layers of GaAs over AlGaAs. The energy gap of GaAs increases upon $\mathrm{Al}$ doping. When GaAs is doped with donors at zero temperature the Fermi level lies higher than the bottom of the conduction band. The electrons bound to donors move into GaAs conduction band and the process stops when some proportion of electrons have moved. The electrons in the inversion layer are two dimensional as shown in Fig. 2.

The average drift velocity of the electron subjected to the electric field is,

$$
V_{d}=-e E \tau / m
$$

where $E$ is the electric field, $m$ is the electron mass and $\tau$ is the mean life time so that the current density is,

where

$$
j=-n e V_{d}=\sigma_{o} E
$$




$$
\sigma_{o}=n e^{2} \tau / m
$$

where $n$ is the electron density. In the presence of a steady magnetic field, the conductivity and resistivity become tensors/matrices,

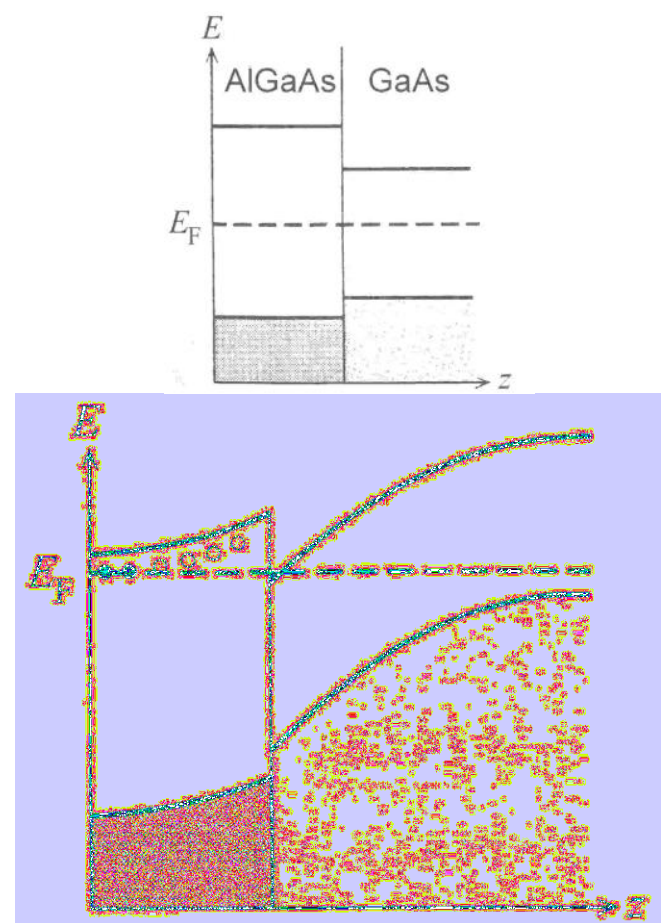

Fig. 2. To make a two-dimensional electron system, layers of AlGaAs are deposited over GaAs.

$$
\begin{gathered}
\sigma=\left(\begin{array}{ll}
\sigma_{x x} & \sigma_{x y} \\
\sigma_{y x} & \sigma_{y y}
\end{array}\right), \\
\rho=\left(\begin{array}{ll}
\rho_{x x} & \rho_{x y} \\
\rho_{y x} & \rho_{y y}
\end{array}\right)
\end{gathered}
$$

We take $x$ and $y$ axes in the 2-dimensional plane, to obtain,

$$
\begin{aligned}
& i_{x}=\sigma_{x x} E_{x}+\sigma_{x y} E_{y}, \\
& i_{y}=\sigma_{y y} E_{x}+\sigma_{y x} E_{y}
\end{aligned}
$$

Owing to the isotropy, $\sigma_{x x}=\sigma_{y y}$ and $\sigma_{x y}=-\sigma_{y x}$. The first one is called the diagonal conductivity and the second one is called the Hall conductivity. The relation between conductivity and resistivity is,

$$
\rho_{x x}=\rho_{y y}=\frac{\sigma_{x x}}{\sigma_{x x}{ }^{2}+\sigma_{x y}{ }^{2}}
$$

for the diagonal resistivity and for the Hall resistivity, we obtain,

$$
\rho_{x y}=-\rho_{y x}=-\frac{\sigma_{x y}}{\sigma_{x x}^{2}+\sigma_{x y}^{2}}
$$

We measure these quantities by connecting various leads as given in Fig. 3 .

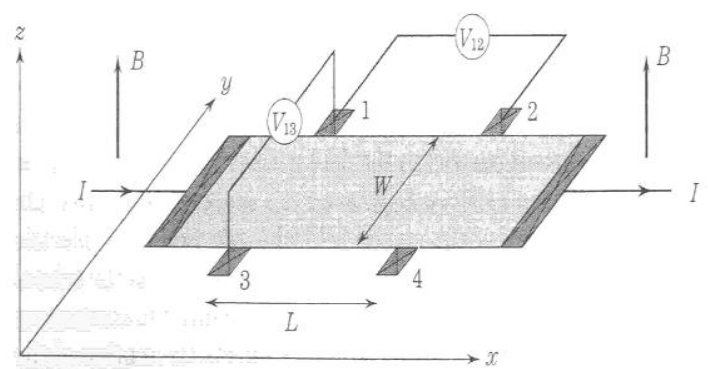

Fig. 3. The sample with the magnetic field B and the current I perpendicular to it.

The wires are connected to measure Hall voltage perpendicular to both I and $\mathrm{B}$ in various directions. $\mathrm{W}$ is the width of the sample and $\mathrm{L}$ is the length.

In the case of homogeneous current in the y direction, $i_{x}=I / W$ and $i_{y}=0$. The electric field is given by, $E_{x}=V_{12} / L$ and $E_{y}=V_{13} / W$ so we have, $\rho_{x x}=V_{12} W / I L$ and $\rho_{y x}=V_{13} / I=R_{H}$. The Hall resistivity in two dimensional electron system is the Hall resistance per unit area. According to the Drude (ordinary non-interacting metals) theory,

$\rho_{x x}=1 / \sigma_{o}=m / n e^{2} \tau$ and $\sigma_{x y}=B / n e c$ in a weak magnetic field. The Hall resistivity is inversely proportional to the electron density and independent of mean scattering life time. In strong magnetic fields, there are new phenomena. Von Klitzing found the plateau in the Hall resistivity which gave the correct value of $h / e^{2}$. One of his plateaus is given in Fig. 4 . We see from the plot that (i) there is a plateau region in which the Hall resistivity remains constant. As the electron density is varied in this region the diagonal resistivity is almost zero. (ii) The value of the Hall resistivity in the plateau regions is exactly equal to $h / e^{2}$ divided by an integer. Therefore, the Hall conductivity $\sigma_{\mathrm{xy}}$ in the plateau region is "quantized" into integer multiples of $e^{2} / h$. This phenomenon was called the "integer quantized Hall effect (IQHE). Since the bending point occurs a little bit earlier than the plateau, the value is uncertain by that much.

\section{QUANTUM HALl EFFECT}

\section{A. Flux Quantization and the Hall Effect.}

Introduction of the flux quantization immediately explains von Klitzing's plateau. We have the Hall resistivity as,

$$
\rho=B / n e c
$$

where $B$ is the magnetic field. According to flux quantization, the field in a certain area, A, is quantized,

$$
B . A=m \varphi_{o}
$$

where the magnetic flux quantum is, $\varphi_{o}=h c / e$. Substitution of (9) into (8) gives the integer quantized Hall effect as,

$$
\rho=\frac{\frac{m h c}{A e}}{\frac{N_{o}}{A} e c}=\frac{m}{N_{o}} \frac{h}{e^{2}}
$$


The quantum Hall effect thus is the quantization of Hall resistivity as,

$$
\rho=\frac{h}{i e^{2}}
$$
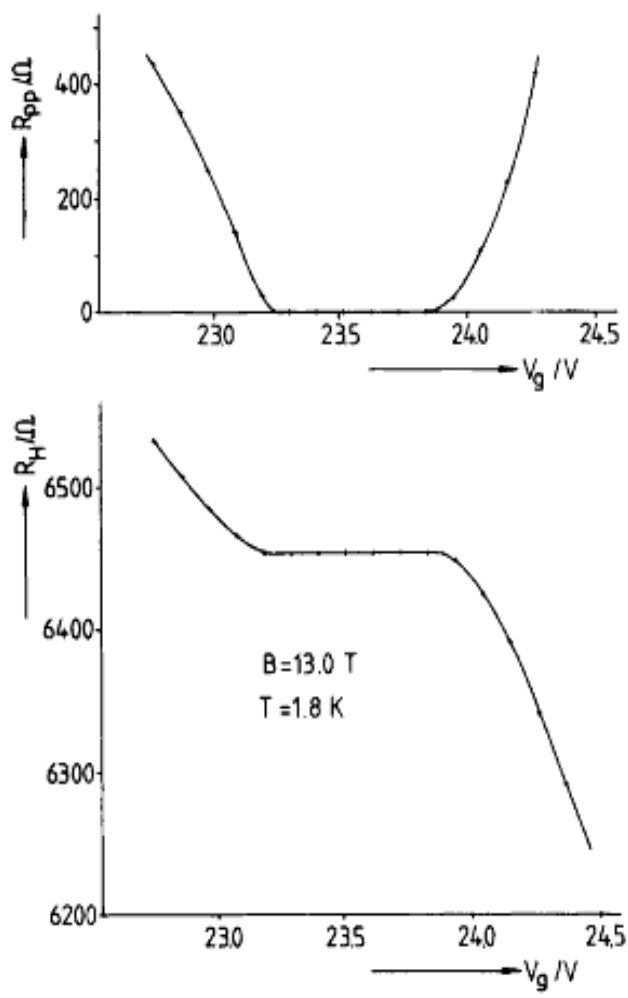

Fig. 4. The Hall resistivity shows plateau at high fields. The picture shows von Klitzing's data at a field of 13 Tesla (13x104Gauss).

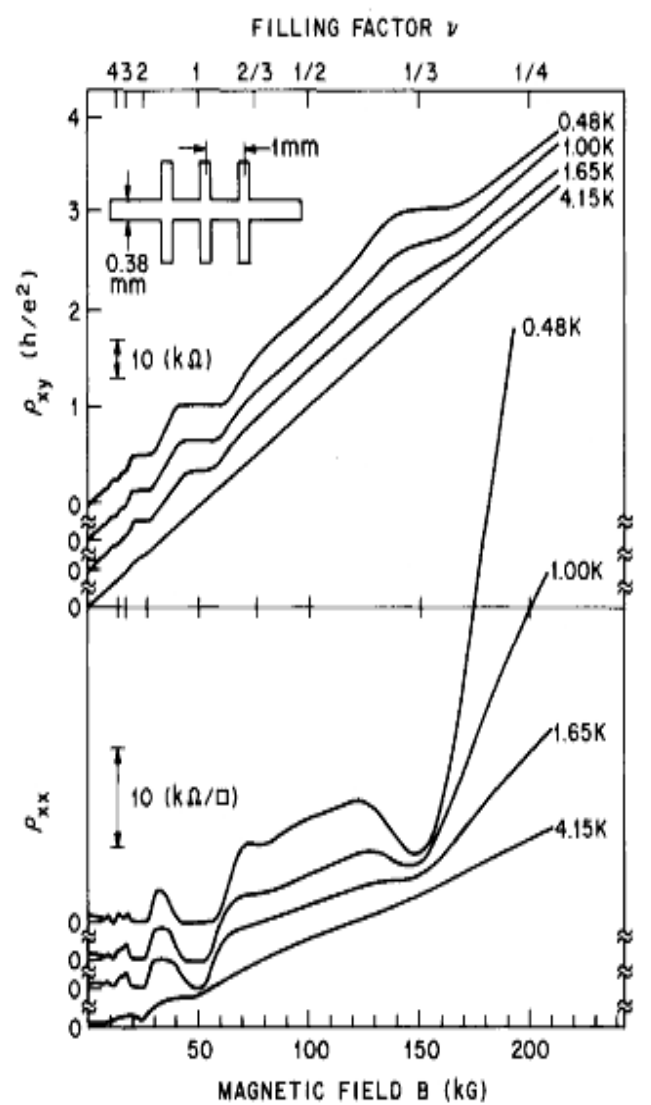

Fig. 5. The data of Hall resistivity showing plateau at $1 / 3$ below a temperature of 1 K. (D.C. Tsui, H.L.Stormer and A.C. Gossard).
Hence the charge of the quasiparticle is ie . Here $\mathrm{i}=$ integer. The charge thus becomes $1 e, 2 e, 3 e, \ldots, \mathrm{i} e, \ldots$ von Klitzing obtained the correct value of the charge for $i=1$. So the value of $\mathrm{h} / \mathrm{e}^{2}$ became "one von Klitzing". When this experiment was repeated with cleaner samples with higher electron mobility, with higher magnetic fields and lower temperatures, it lead to the discovery of $i=1 / 3$ plateau which gave birth to the fractional charge. We show the data of Tsui, Stormer and Gossard in Fig. 5 with plateau at $1 / 3$. Since this fractional value occurred in the middle of various highly degenerate Landau levels, where no gap is apparent, the observation could not be explained by the experimentalists by using the non-interacting quantum mechanical theory. It was thought that the observation is a result of the many-body effects of electron interactions. Subsequent experimental work showed a lot many more plateaus which are displayed in Fig.6. We will see that there is no need of interaction to explain the plateau at $1 / 3$.

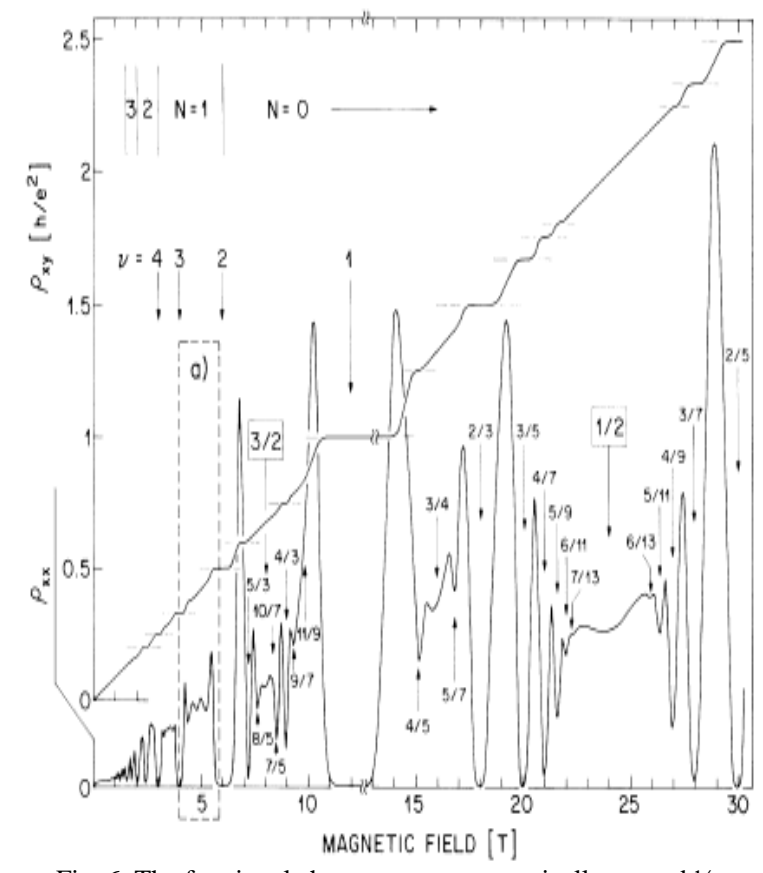

Fig. 6. The fractional charges seen symmetrically around 1/2.

\section{B. Laughlin's Theory}

Laughlin made the first efforts to explain the quantum Hall effect. The flux quantization was immediately found to explain at least the integer quantized Hall effect. Subsequently, Laughlin started from first principles using the Hamiltonian,

$H=\sum_{j}\left\{(\hbar / i) \nabla_{j}-\left.(e / c) \vec{A}_{j}\right|^{2}+V\left(z_{j}\right)\right\}+\sum_{j>k} \frac{e^{2}}{\left|z_{j}-z_{k}\right|}$

where $j$ and $k$ sum over $\mathrm{N}$ particles and $\mathrm{V}$ is the potential due to nuclei. The repulsive Coulomb interactions can produce the plateaus only when flux quantization is considered. A trial wave function of the form given below,

$$
\left|\Psi\left(z_{1}, \ldots, z_{N}\right)\right|^{2}=e^{-\beta(\phi+\gamma)}
$$


with $\beta=1 / m$,

$$
\phi=-2 m^{2} \sum_{j<k} \ln \left|z_{j}-z_{k}\right|+m / 2 \sum_{\ell}^{N}\left|z_{\ell}\right|^{2}
$$

and $\gamma$ is a multicenter integral, to solve the Schrodinger equation to find the ground state. Laughlin obtained the approximate energy expression in terms of $\mathrm{m}$ as well as computed exactly. By using "incompressibility" the charge of the particles is fixed at $1 / 3$ and $1 / 5$. Unfortunately, there is area in the flux quantization which must also be fixed, otherwise the charge will leak. Laughlin also shared the 1998 Nobel prize with Tsui and Stormer but it is now believed that Laughlin's wave function is not relevant to explain the data.

\section{Shrivastava's Theory}

We consider that electrons have spin as well as the orbital angular momentum so that,

$$
g_{j} j=g_{s} \vec{s}+g_{l} \vec{l}=\frac{1}{2}\left(g_{l}+g_{s}\right) \vec{j}+\frac{1}{2}\left(g_{l}-g_{s}\right)(\vec{l}-\vec{s})
$$

Multiplying both sides by $j=l+s$ and taking eigen values,

$$
g_{j} j(j+1)=
$$

$1 / 2\left(g_{l}+g_{s}\right) j(j+1)+1 / 2\left(g_{l}-g_{s}\right)[(\vec{l}(l+1)-s(s+1)]$

Substituting $s=1 / 2$ and $j=l \pm(1 / 2)$ we get,

$$
g_{j}=g_{l} \pm \frac{g_{s}-g_{l}}{2 l+1}
$$

For $g_{\mathrm{s}}=2, g_{1}=1$, we find,

$$
g_{ \pm}=1 \pm \frac{1}{2 l+1}
$$

The equation (17) has both the signs for the spin. The cyclotron frequency is,

$$
\omega=\frac{e B}{m c}
$$

From the charge, e in the cyclotron frequency, we generate the charge of a particle. It is also possible to obtain the charge from the e in Bohr magneton. Corresponding to the cyclotron frequency, the voltage along y direction is,

$$
\hbar \omega=\mathrm{e} V_{y} .
$$

or,

$\hbar \frac{e B}{m c}=e V_{y}$. Multiplying this expression by $e / h$, we get,

$$
\frac{e^{2} B}{2 \pi m c}=\frac{e^{2}}{h} V_{y}
$$

which is the current in $\mathrm{x}$ direction, so that the resistivity,

$$
\rho_{x y}=\frac{h}{e^{2}}
$$

The $S_{z}=1 / 2$, and the energy in a magnetic field is $g \mu_{\mathrm{B}} \mathrm{H} . \mathrm{S}$, so correcting B in the cyclotron frequency we find,

$$
\mathrm{I}_{\mathrm{x}}=\frac{1}{2} g \frac{e^{2} B}{2 \pi m c}=\frac{1}{2} g \frac{e^{2} V_{y}}{h}
$$

For $l=0, g=2$,

$$
\mathrm{I}_{\mathrm{x}}=\frac{e^{2}}{h} V_{y}
$$

which describes the quantized current correctly for $v=1$. From the above equations we have $v=\frac{1}{2} g_{ \pm}$which gives the filling factor, one for + sign and the other for - sign as in (18). For $l=0$, we obtain $(1 / 2) g_{+}=1$ and $(1 / 2) g_{-}=0$ and other values as given in Table 1.The Landau levels are introduced by multiplying the above values by $\mathrm{n}$ so that $v=n\left(\frac{1}{2} g_{ \pm}\right)$so we can multiply the tabulated values by an integer when needed. The values of $\rho_{x x}$ and $\rho_{x y}$ for a single interface of $\mathrm{GaAs} / \mathrm{AlGaAs}$ have been measured by Willet et al at $150 \mathrm{mK}$. The values predicted in the Table I are exactly the same as in Willet's experimental data shown in Fig. 7. The values shown in the figure occur in two sets,

The values $2 / 5,3 / 7,4 / 9,5 / 11,6 / 13, \ldots$ etc. and $2 / 3,3 / 5$, $4 / 7,5 / 9,6 / 11$ etc. The predicted values in the table are the same as in the experimental data. Using the table 1, when we multiply the values by $n$ we can interpret all of the experimentally measured values. The columns of Table I belong to two signs of spin, one belonging to $+1 / 2$ and the other to $-1 / 2$. For the cyclotron frequency $\hbar \omega_{c}=g \mu_{B} B$ where $\mu_{\mathrm{B}}=\mathrm{e} \hbar / 2 \mathrm{mc}$ is the Bohr magneton. Therefore, $(1 / 2) \mathrm{g}_{ \pm}$can be considered to be the effective charge, $e_{e f f}=\frac{1}{2} g e=v e$. In Table I, we see two series, $v_{-}=\frac{l}{2 l+1}$ and $v_{+}=\frac{l+1}{2 l+1}$ which can be used to explain the high Landau levels easily.

Eisenstein et al have found that for the higher values of the Landau level quantum number, $\mathrm{n}$, the number of fractions observed are much less than at the lowest Landau level. At the magnetic field of 4 or 5 Tesla only a small number of fractions are observed, the strongest ones are at: $8 / 3,5 / 2$ and $7 / 3$. The series $l /(2 l+1)$ is the particle-hole conjugate of $(l+1) /(2 l+1)$. For $l=7$, two values, $7 / 15$ and $8 / 15$ are predicted and for $l=\infty$ the value is $1 / 2$. When the same particle occurs in different levels its charge remains unchanged. We can multiply the values by $n=5$ so that the predicted values of $1 / 2,7 / 15$ and $8 / 15$ become $5 / 2,7 / 3$ and $8 / 3$. These predicted values are exactly the same as those observed experimentally by Eisenstein et al. Thus, $7 / 3$ is the particle-hole conjugate of $8 / 3$ as seen in Table II for $n=5$. 
TABLE I: THE PRINCIPAL FRACTIONS OBTAINED FROM THE TWO SERIES

\begin{tabular}{|l|l|l|}
\hline$l$ & $(1 / 2) g_{-}=l /(2 l+1)$ & $(1 / 2) g_{+}=(l+1) /(2 l+1)$ \\
\hline 0 & 0 & 1 \\
\hline 1 & $1 / 3$ & $2 / 3$ \\
\hline 2 & $2 / 5$ & $3 / 5$ \\
\hline 3 & $3 / 7$ & $4 / 7$ \\
\hline 4 & $4 / 9$ & $5 / 9$ \\
\hline 5 & $5 / 11$ & $6 / 11$ \\
\hline 6 & $6 / 13$ & $7 / 13$ \\
\hline$\infty$ & $1 / 2$ & $1 / 2$ \\
\hline
\end{tabular}

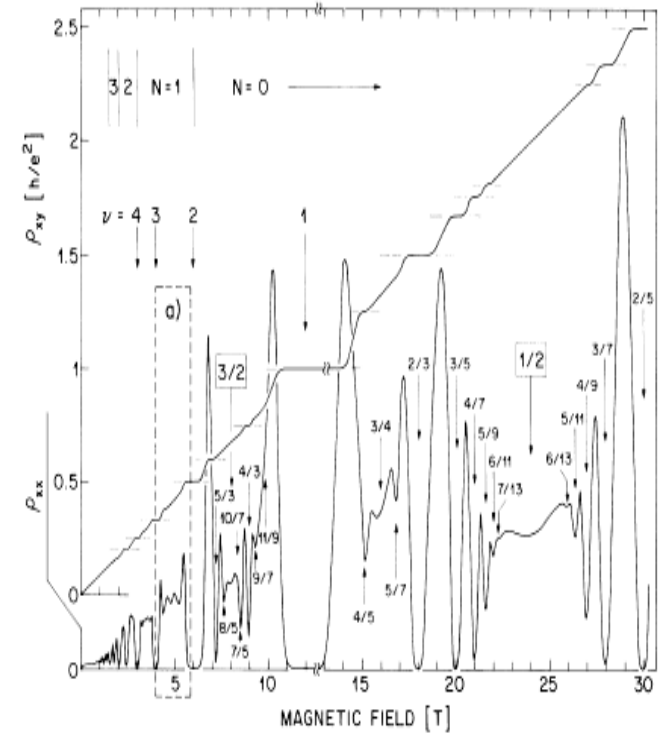

Fig. 7. The experimental data on Hall resistivity showing fractions of charges.

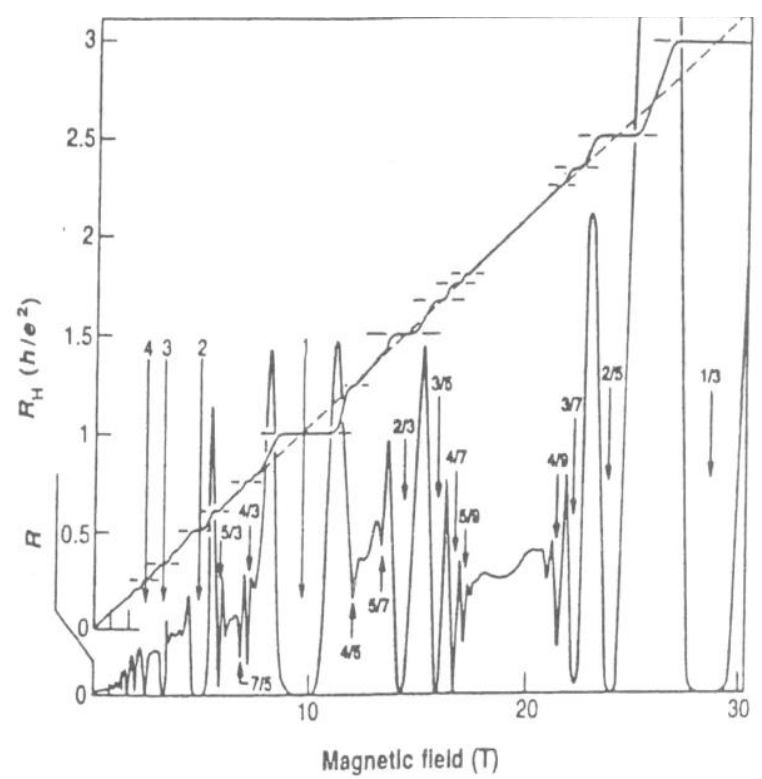

Fig. 8. Hall effect data showing plateau at $1 / 3$. The observed series of charges are exactly the same as in Table I.

For a long time only odd denominators were reported which show that even denominators are weak. After that even denominators as well as even numerators with odd denominators are found. We go back to the same formula, $\frac{l+\frac{1}{2} \pm s}{2 l+1}$. When $s=1, l=0$, it is $3 / 2$ for + sign. Hence for electron clusters or pairs the fraction has even denominator. Since the number of particles is larger than one its probability becomes small so these plateaus are weak but the same theory explains the even denominators. There is a limiting value of the series which also gives $1 / 2$. One can introduce a Fermi surface at $n / 2$, Thus $1 / 2,2 / 2,3 / 2,4 / 2,5 / 2,6 / 2$ and $7 / 2$ are predicted which are observed by Yeh et al. [8] as shown in Fig. 9.

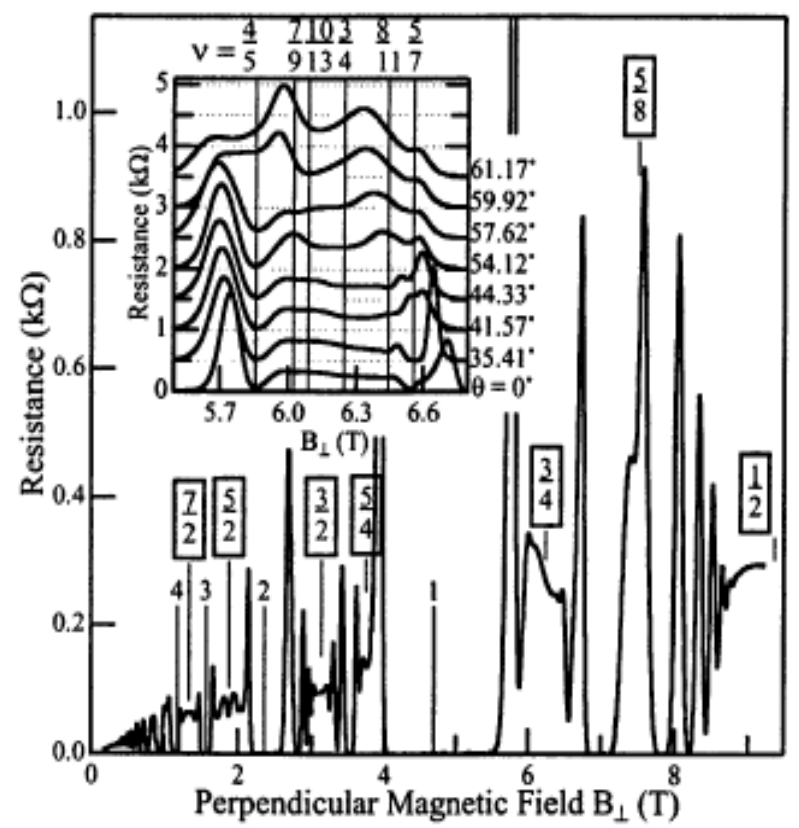

Fig. 9. The predicted $n / 2$ is observed in this experimental data.

TABLE II: EFFECT OF N THE LANDAU LEVEL NUMBER

\begin{tabular}{|l|l|l|l|l|}
\hline$l$ & $l /(2 l+1)$ & $(l+1) /(2 l+1)$ & $n l /(2 l+1)$ & $n(l+1) /(2 l+1)$ \\
\hline$\infty$ & $1 / 2$ & $1 / 2$ & $5 / 2$ & $5 / 2$ \\
\hline 7 & $7 / 15$ & $8 / 15$ & $7 / 3$ & $8 / 3$ \\
\hline
\end{tabular}

It has been reported by Yeh et al. [8] that the effective mass and $\mathrm{g}$ factor of some of the fractions are equal to those of others. We have shown that the effective mass can be equal only when the two quasiparticles are particle-hole conjugates. The particle-hole conjugates should obey the following relation,

$$
v_{p}+v_{h}=1
$$

The values given in Table I always obey this relation.

\section{Half filled Landau Level}

The $l=\infty$ in the two series produces,

$$
\begin{aligned}
& \lim _{l \rightarrow \infty} v_{+}=\frac{1}{2}(+) \\
& \lim _{l \rightarrow \infty} v_{-}=\frac{1}{2}(-)
\end{aligned}
$$

One $1 / 2$ comes from the right and the other from the left when magnetic field is varied, i.e., one while increasing the field and the other while reducing. One can go from $1 / 3$ to $1 / 2$ by reducing the field while from $2 / 3$ to $1 / 2$ is obtained by increasing the field. We can go from $1 / 3$ to $3 / 5$ by reversing the spin and increasing the $l$. Similarly from $2 / 3$ to $2 / 5$ by reducing the spin and increasing $l$. In this way angular momentum is conserved. One of the $1 / 2$ values is like an electron (1/2)A and the other is like a hole, (1/2)B, (A for + series and B for - series). Since the electron and the hole are separated by a distance, this state is compressible. When we 
multiply this result by $\mathrm{n}$, the Landau level quantum number, we obtain: $1 / 2,2 / 2,3 / 2,4 / 2,5 / 2, \ldots$, which are in agreement with data.

\section{E. Effective Charge}

We discussed the Laughlin's wave function earlier. Here the repulsive Coulomb interactions can not give rise to a fractional charge of $1 / 3$. It is first assumed on the basis of experimental data and then substituted in the theory. Laughlin's charge is independent of spin but in our theory it depends. In Laughlin's theory a particle of charge $1 / 3$ is produced but in our theory, splitting occurs in fractions of $1 / 3$ and $2 / 3$, etc.

\section{Laughlin's 1/3 charge.}

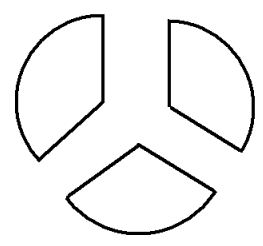

\section{Shrivastava's theory.}

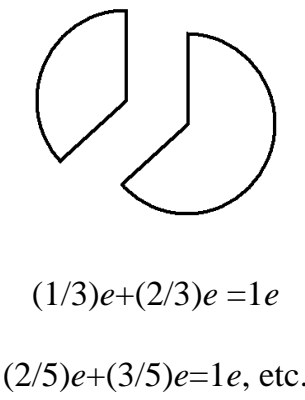

Fig. 10. There is a difference between Laughlin and our theories. The fractionalization in Laughlin's theory is independent of spin. Whereas in our theory spin plays an important role in determining the fraction.

\section{F. Dirac Equation}

The basic idea of Dirac equation is to have space-time symmetry and the constancy of velocity of light, so that instead of $p^{2} / 2 m$, the kinetic energy appears as $c \alpha . p$ and the wave equation becomes,

$$
\left(c \alpha \cdot p+\beta m c^{2}\right) \Psi(x, t)=i \hbar \frac{\partial}{\partial t} \Psi(x, t)
$$

The free particle solutions of which are,

$$
E_{ \pm}= \pm\left(c^{2} p^{2}+m^{2} c^{4}\right)^{1 / 2}
$$

This equation gives the correct magnetic moments for the proton as well as for the neutron subject to using the mass of the respective particle and an appropriate $g$ value. In the case of electron Lande's formula,

$$
g=1+\frac{j(j+1)-l(l+1)+s(s+1)}{2 j(j+1)}
$$

with positive spin is used. In our case, the effective charge and hence the magnetic moment is determined by the $\mathrm{g}$ values. Hence, our method of defining the charge of the electron is the same as that for the magnetic moments of proton and neutron.

\section{G. Shubnikov-de Haas Effect}

At low temperatures, the integration over the Fermi distributon leads to $x / \sinh x$

Type expression which is called Dingle's formula. The spin symmetry is found to modify this formula which determines the oscillation amplitude of resistivity as a function of magnetic field, called the Shubnikov-de Haas effect. Our theory introduces the effective charge so that the cyclotron frequency gets fractionalized resulting into $\mathrm{m} / \mathrm{v}_{ \pm}$ which for $v_{ \pm}=1$ becomes $m$, the electron mass. Thus, we have taken into account, the spin-charge fractions, to obtain the correct mass. For example, at certain magnetic field $1.5 \mathrm{~m}$ is found instead of $\mathrm{m}$. The mass of the electron relative to band value as a function of carrier density deduced from Shubnikov-de Haas effect in GaAs/AlGaAs heterostructures is shown in the Fig. 11. The factors 1 and $2 / 3$ are found to arise from the spin-charge effect of Shrivastava. The experimental data is obtained from Tan, Stormer et al. [9 ]. The dashed line is found by Kwon et al on the basis of small self energy corrections due to many body perturbative interactions. The factors of 1 and 2/3 in the mass are found by us. The mass of the free electron is $m_{e}$ and the screening radius is deduced from the density, $\mathrm{n}$, per unit area. We find that $\mathrm{m} / \mathrm{v}_{ \pm}$occurs in place of $\mathrm{m}$ for the mass of the electron in the Shubnikov-de Haas ( $\mathrm{SdH}$ ) effect. In fact, many other fractions of the mass of the electron given in Table 1, become allowed so that the electron really "falls apart". The oscillations due to flux quantization allow the measurement of $\mathrm{m} / \mathrm{h}^{2}$. The flux quantization in the Shubnikov-de Haas effect leads to "quantized Shubnikov-de Haas effect". Therefore, we observe consequences of the effect of flux quantization on the Shubnikov-de Haas oscillations. There are zeroes in the resistivity at certain fields. There is a spin-charge effect so that the spin flip corresponds to a change in the charge.

The Shubnikov-de Haas effect uses quantization of Landau levels but not the flux quantization. Hence, we find that there is a "quantized Shubnikov-de Haas effect" which measures the $\mathrm{m} / \mathrm{h}^{2}$. We find that when fractional values of $v_{ \pm}$ are taken into account, the mass of the electron, equal to band mass in GaAs/AlGaAs is obtained. When magnetic field is varied, the different values of $n$ cross the Fermi energy at different fields resulting into oscillations in the resistivity as a function of magnetic field. The oscillating resistivity is given by, resistivity is given by,

$$
\begin{aligned}
& \delta \rho_{x x}(\varepsilon)_{ \pm}=\rho_{o} \sum_{p} \gamma_{t h} c_{\alpha, \beta} \exp \left(-\frac{p \pi}{v_{ \pm} \omega_{c} \tau_{q}}\right) c(\varepsilon) \\
& \text { where } \\
& \gamma_{t h}=\frac{2 \pi^{2} p k_{B} T /\left(\hbar \omega_{c} v_{ \pm}\right)}{\sinh \left[2 \pi^{2} p k_{B} T /\left(\hbar \omega_{c} v_{ \pm}\right)\right]} \\
& c(\varepsilon)=\cos \left[2 \pi p\left(\frac{\varepsilon}{v_{ \pm} \hbar \omega_{c}}-\frac{1}{2}\right)\right]
\end{aligned}
$$


The cosine factor also leads to zero resistivity when ever,

$$
2 \pi p\left(\frac{\varepsilon}{v_{ \pm} \hbar \omega_{c}}-\frac{1}{2}\right)=\frac{\pi}{2}
$$

so that the resistivity vanishes when $B$ satisfies the above formula. We introduce the flux quantization so that the exponential factor in (30) becomes,

$$
\exp \left(-\frac{P \pi m c}{v_{ \pm} \tau_{c} e B}\right)=\exp \left(-\frac{\pi m A}{v_{ \pm} \tau_{c} h}\right)
$$

so that $m / h$ will be measured from the oscillations. Introducing the flux quantization in the argument of Sinh factor, we obtain,

$$
\hbar v_{ \pm} \omega_{c}=\frac{\hbar v_{ \pm} n_{2} h}{m A}
$$

which does not have the charge but measures $\mathrm{m} / \mathrm{h}$. In the experiments the factor measured is $\mathrm{m}^{*} g^{*} / \mathrm{n}$ so it is clear that the mass and g get mixed.

\section{H. Spin-Charge Locking}

The charge of the electron may be described by matrices just as the angular momentum is. When the spin is aligned along the charge, such as $\mathrm{s}_{\mathrm{x}}$ parallel to $\mathrm{e}_{\mathrm{x}}$, the arrangement is called the spin-charge locking. Taking our effective charge expression for $\mathrm{e}_{\mathrm{eff}} / \mathrm{e}=(1 / 2) \mathrm{g}_{ \pm}$we find the dot product of spin and charge to find,

$$
\begin{aligned}
& e^{*} \cdot s^{\prime}=(2 l+1)^{-1}\left(l+\frac{1}{2} \pm s\right) \cdot s^{\prime} \\
& =\frac{1}{2 l+1}\left[\left(l \cdot s^{\prime}\right)+(1) \cdot s^{\prime}+s \cdot s^{\prime}\right]
\end{aligned}
$$

which produces spin-orbit and spin-spin interactions and there is spin divided by $2 l+1$. That is what makes it difficult to detect this type of effect.

We have thus found the correct explanation of the experimentally observed quantum Hall effect. We find that angular momentum gives rise to fractional charge. Therefore, there is a spin-charge effect, i.e., under high magnetic fields, the spin determines the charge the Lande's formula is replaced by a linear formula and Landau levels are modified [11-24]. Laughlin's wave function is not the interpretation of quantum Hall effect [25].

\section{HELICITY IN QUANTUM HALl EFFECT}

We look at the charge of the electrons in the quantum Hall effect as $e^{*}=(1 / 2) g_{ \pm} e$. For $l=0$,

$$
e^{*} / e=\frac{1}{2} \pm s
$$

Hence we predict particles of charge 0 and 1 . The particle of charge zero uses the negative sign and hence its helicity is negative. The particle of charge 1 uses the positive sign. Hence its helicity is positive. The particles occur in pairs. Hence particle is defined by positive helicity and its charge is equal to that of the electron charge. The particle of charge zero has negative helicity and hence it is the antiparticle analog of the electron of charge 1 .

Particle: helicity positive, charge $=1$,

Antiparticle: helicity negative, charge $=0$.

For $l=1, s=1 / 2, e^{*}=1 / 3$ with negative helicity and $e^{*=2 / 3}$ for positive helicity. These values are exactly the same as observed in the experimental data. Hence we can determine the helicities of all of the plateaus. The particle of charge $1 / 3$ is left handed. Li et al. [10] have performed the experimental measurements in AlGaAs from which we observe that the plateaus occur at, 1/5, 2/9, 2/3, 3/5, 2/5, 1/3, 5/3, 4/3, 4/7, $7 / 11,3 / 7$ and $63 / 100$.

We explain all of these values and determine the helicity in each case.

1) For $2 l+1=5, l=2, s=1 / 2$, the principal fractions occur at $l /(2 l+1)$ and $(l+1) /(2 l+1)$ so that $2 / 5$ has negative helicity and $3 / 5$ has positive helicity. The resonance occurs at $3 / 5-2 / 5=1 / 5$. This explains the plateau at $1 / 5$ as a "resonance". It belongs to mixed helicity. It can rotate and the velocity can change sign during rotation such that $3 / 5$ can become $2 / 5$ and $2 / 5$ can become $3 / 5$ so that $3 / 5+2 / 5=2 / 5+3 / 5=1$ becomes a particle of charge 1 with mixed helicity and $3 / 5-2 / 5=1 / 5$ becomes $2 / 5-3 / 5=-1 / 5$ which is a hole in the band theory. The hole has the charge of opposite sign compared with that of the electron. Thus we have particles of mixed helicities with charge $\pm 1 / 5$ as well as $2 / 5$ and $3 / 5$.

2) The charge $2 / 9$ has the denominator of $2 l+1=9$. Hence, $l$ $=4$. The $l /(2 l+1)=4 / 9$ and $(l+1) /(2 l+1)=5 / 9$. The resonance occurs at $5 / 9-4 / 9=1 / 9$ and $4 / 9$ has negative helicity whereas $5 / 9$ has positive helicity. The charge $1 / 9$ has mixed helicity and the two particle state at $1 / 9+1 / 9=2 / 9$ has mixed helicity.

3) The state $2 / 3$ has $l=1$ and $(l+1) /(2 l+1)=2 / 3$ has positive helicity and $l /(2 l+1)=1 / 3$ has negative helicity.

4) The states $2 / 5$ and $3 / 5$ are the $l=2, s=1 / 2$ with $l /(2 l+1)=2 / 5$ for negative helicity and $(l+1) /(2 l+1)=3 / 5$ for positive helicity.

5) The state $5 / 3>1$ is not due to the principal fractions. It requires that a contribution should come from the bosonic character of the Landau levels. It is possible to make $5 / 3$ as $1 / 3+1 / 3+1 / 3+1 / 3+1 / 3=5 / 3$ which is of negative helicity. It is also possible to make $2 / 3+2 / 3+1 / 3=5 / 3$ which is of mixed helicity. It can rotate to make $2 / 3+1 / 3+2 / 3=5 / 3$ which is an example of "reversed helicity". It is possible to make a state $1 / 3+1 / 3+2 / 3=4 / 3$ which is a case of mixed helicity. If $2 / 3+2 / 3+1 / 3=5 / 3$ is a particle then $1 / 3+1 / 3+2 / 3=4 / 3$ is the antiparticle analog of $5 / 3$. The $4 / 3$ as well as $5 / 3$ are degenerate because there is more than one way of making them. The state $2 / 3+2 / 3=4 / 3$ as well as $1 / 3+1 / 3+2 / 3=4 / 3$ are degenerate.

6) The denominator $2 l+1=7$ is made from $l=3$. Hence, the principal value for negative helicity $l /(2 l+1)=3 / 7$ and 
for positive helicity, $(l+1) /(2 l+1)=4 / 7$.

7) For $2 l+1=11, l=5$. Hence the principal value for positive helicity is $6 / 11$ and for negative helicity it is $5 / 11$. The resonance occurs at $6 / 11-5 / 11=1 / 11$. The sum process gives $6 / 11+1 / 11=7 / 11$ which is of mixed helicity.

8) The value of $63 / 100$ can be written as $(1 / 2)(63 / 50)=(1 / 2)(1 / 2)(63 / 25)$. For a large $l=12,2$ $l+1=25$. Hence $12 / 25$ and $13 / 25$ are the particle-antiparticle conjugates. The $63 / 25$ is the sum as $50 / 25+13 / 25=2+13 / 25$ which is above the second Landau level. The flux quantization occurs at n'hc/e. For $\mathrm{n}^{\prime}=2$ the charge reduces to $1 / 2$ as $\mathrm{hc} /(\mathrm{e} / 2)$ so that we can reduce the charge to $1 / 2$ of its value. Hence $63 / 25$ becomes $63 / 50$. For $l+(1 / 2) \pm s=63, l=12$ we have $\pm \mathrm{s}=63-12-(1 / 2)=101 / 2$ which is possible in a cluster of electrons. For Landau levels, for $\mathrm{g}_{1}=0, \mathrm{n}_{2}=0$,

$$
\frac{1}{2} g_{2}\left(n_{2}+\frac{1}{2}\right)=\frac{1}{4} g_{2}
$$

Hence a factor of $1 / 4$ comes so that $63 / 25$ becomes $63 / 100$. The Landau levels with suitable quantum numbers with positive helicity explain the value of 63/100. In this way, the "principal fractions", the resonances, the sum process as well as clustering explain the experimental data of AlGaAs.

9) For $s=0,(1 / 2) g=1 / 2$ so that $e^{* / e}=1 / 2$ for both helicities. For $s=0$, the concept of particle and antiparticle disappears and the particles are said to become "Majorana type".

\section{BREMSSTRAHLUNG}

Bremsen means to brake (slow down) and Strahlung means radiation, i.e., radiation produced by slowing down the electrons. The electron with spin $1 / 2$ and energy $E_{2}$ slows down to a lower energy $E_{1}$ and the energy is conserved by emission of radiation. There is an electron of energy $E_{2}$ in the initial state in the initial state and there is an electron with energy $\mathrm{E}_{1}$ as well as a photon in the final state, $E_{2}=E_{1}+h v$. In the quantum Hall effect, a particle of charge 1/3 travelling with negative helicity (left handed) emits a photon (unpolarized) and a particle of charge $1 / 3$ continues to propagate. It is possible that a particle of charge $1 / 3$ emits a particle of zero charge and a particle of $1 / 3$ charge. A particle of charge $2 / 3$ moving with positive helicity can slow down to emit two particles one of charge $2 / 3$ and the other of charge zero.

\section{CONCLUSION}

The quantum Hall effect first observed by von Klitzing et al had no theory at all and the origin of fractions has not been explained by them. In particular they have not explained the fractional charge at which the plateaux occur. We have explained the fractions from our original theory [7] and found that Lande's as well as Landau's theories require modifications. The role of angular momentum in the understanding of the quantum Hall effect is quite clearly demonstrated.

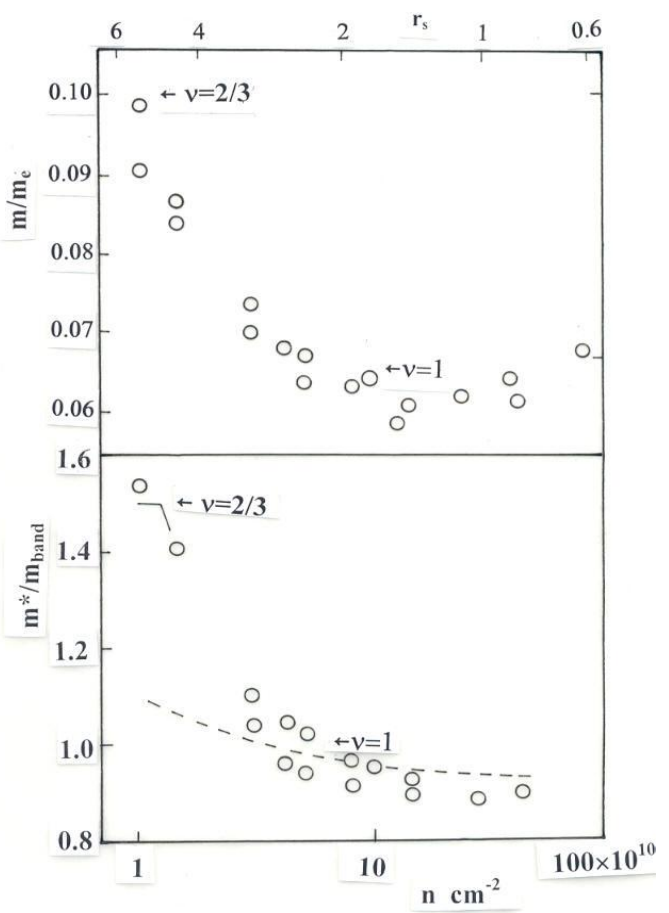

Fig. 11. The mass of the electron deduced from the amplitude of oscillations as affected by our charge factors.

\section{REFERENCES}

[1] E. Hall, "On a new action of the magnet on electric currents," American J. Maths, vol. 2, pp. 287-292, 1879.

[2] T. Englert and K. V. Klitzing, Surf. Sci., vol. 73, no. 70, 1978.

[3] K. V. Klitzing, G. Dorda, and M. Pepper, "New method for high-accuracy determination of the fine structure constant," Phys. Rev. Lett., vol. 45, pp. 494-497, 11 August 1980

[4] D. C. Tsui, H. L. Stormer, and A. C. Gossard, "Two-dimensional magnetotransport in the extreme quantum limit," Phys. Rev. Lett., vol. 48, pp. 1559-1562, 1982.

[5] R. Willett, J. P. Eisenstein, H. L. Stormer, D. C. Tsui, A. C. Gossard, and J. H. English, "Observation of an even-denominator quantum number in the fractional quantum Hall effect," Phys. Rev. Lett., vol. 59 , pp. 1776-1779, 1987.

[6] R. B. Laughlin, "Anomalous quantum Hall effect: An incompressible quantum fluid with fractionally charged excitations," Phys. Rev. Lett., vol. 50 , pp. 1395-1398, 1983.

[7] K. N. Shrivastava, "Rational numbers of the fractionally quantized Hall effect,” Phys. Lett. A, vol. 113, pp. 435-436, 1986.

[8] A. S. Yeh, H. L. Stormer, D. C. Tsui, L. N. Pfeiffer, K. W. Baldwin, K. W. West, Phys. Rev. Lett., vol. 62, pp. 592, 1999.

[9] Y. W. Tan, J. Zhu, H. L. Stormer, L. N. Pfeiffer, K. W. Baldwin, and K W. West, "Measurement of the density-dependent many-body electron mass in two dimensional GaAs/AlGaAs heterostructures," Phys. Rev. Lett., vol. 94, pp. 016405-1-4, 2005.

[10] W. Li et al., Phys. Rev. Lett., vol. 105, pp. 076803, 2010.

[11] K. N. Shrivastava, "Negative-spin quasiparticles in quantum Hall effect," Phys. Lett. A, vol. 326, pp. 469-472, 2004.

[12] K. N. Shrivastava, "Particle-hole symmetry in quantum Hall effect," Mod. Phys. Lett. B, vol. 13, pp. 1087-1090, 1999.

[13] K. N. Shrivastava, "Theory of quantum Hall effect with high Landau levels," Mod. Phys. Lett. B, vol. 14, pp. 1009-1013, 2000.

[14] K. N. Shrivastava, Frontiers of fundamental physics $4,1^{\text {st }}$ ed. B. G. Sidharth, N.Y.: Academic/Plenum Pub., 2001, pp.235-249.

[15] K. N. Shrivastava, Introduction to quantum Hall effect, New York: Nova Sci. Pub., 2002.

[16] K. N. Shrivastava, Quantum Hall effect: Expressions, New York: Nova Science Pub., 2005.

[17] K. N. Shrivastava, "Additional Dirac matrix in quantum Hall effect," in Proc. AIP, vol. 1017, pp. 47-56, 2008.

[18] K. N. Shrivastava, "The theory of the quantum Hall effect," in Proc. AIP, vol. 1017, pp. 422-428, 2008.

[19] N. A. Zabidi, H. A. Kassim, and K. N. Shrivastava, "DFT calculation of band structure of carbon chain pulled from grapheme," in Proc. AIP, vol. 1250, pp. 241-244, 2010. 
[20] N. A. Rosli, H. A. Kassim, and K. N. Shrivastava, "Graphene infrared spectroscopy: DFT vibrational frequencies," in Proc. AIP, vol. 1250 , pp. 269-272, 2010.

[21] V. R. Devi and K. N. Shrivastava, "DFT calculation of vibrational frequencies of GaAs and the Raman spectra," Spectrochimica Acta A, vol. 95, pp. 172-176, 2012.

[22] K. N. Shrivastava, "The Lande's g value in quantum dots in AlGaAs," International Journal of Nanoscience, World Sci., vol. 10, pp. 507-514, 2011.

[23] K. N. Shrivastava, "The quantum Hall effect," in Proc. AIP, vol. 1482, pp. 335-339, 2012.

[24] M. M. A. Ali and K. N. Shrivastava, "Symmetry properties of Laughlin's wave function and related states," in Proc. AIP, vol. 1482, pp. 43-46, 2012.

[25] K. N. Shrivastava, "Laughlin's wave function and angular momentum," International Journal of Modern Physics B, vol. 25, pp. 1301-1357, 2011.

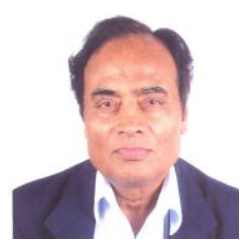

Keshav N. Shrivastava was born on July 11, 1943 He obtained his B.Sc. degree from the Agra University in 1961, M.Sc. degree from the University of Allahabad in 1963, Ph.D. from the Indian Institute of Technology Kanpur in 1966 and D.Sc. from Calcutta University in 1980.

He worked at the Clark University during 1966-68, at the Harvard University during the summer of 1968, at the Montana State University during 1968-69, and at the University of Nottingham during 1969-70. He was Associate Professor at the Himachal Pradesh University Simla, India during 1970-74. He worked at the University of California Santa Barbara during 1974-75 and at the State University Utrecht during 1975-76. He was Professor at the University of Hyderabad where he worked from 1978 till 2005. He worked in Tohoku University during 1999-2000. He visited the University of Zurich for short times during 1984, 1985, 1988 and 1999. He worked in the University of Houston in the summer of 1990. He visited the Royal Institute of Technology Stockholm and the University of Uppsala during 1994. He visited the Technical University of Vienna, Austria in the summer of 1995 . He visited the Princeton University and the University of Cincinnati during 2003. He was Professor in the University of Malaya Kuala Lumpur during 2005-2011. He is affiliated with the University of Hyderabad, India. He has worked in electron spin resonance, spin-phonon interaction, relaxation, Moessbauer effect, exchange interaction, superconductivity and the quantum Hall effect. He discovered the flux quantized energy levels in superconductors, the spin dependent flux and the correct theory of the quantum Hall effect. He has published more than 227 papers in journals and he is the author of the following books. (i) Superconductivity: Elementary Topics, World Scientific Singapore 2000. (ii) Introduction to Quantum Hall effect, Nova Sci. Pub N.Y. 2002 and (iii) The quantum Hall effect: Expressions, Nova Sci. N.Y. 2005.

Prof. Shrivastava is a Fellow of the Institute of Physics London, Fellow of the National Academy of Sciences India and member of the American Physical Society. 